MATEC Web of Conferences 3, 01042 (2013)

DOI: $10.1051 /$ matecconf/20130301042

(C) Owned by the authors, published by EDP Sciences, 2013

\title{
SLE for the carbon dioxide +trans-1,3,3,3-tetrafluoropropene
}

\author{
G. Di Nicola ${ }^{1}$, I. Cacciatore ${ }^{1}$, M. Pierantozzi ${ }^{1}$, and A. Arteconi ${ }^{2}$ \\ ${ }^{1}$ DIISM, Università Politecnica delle Marche, Ancona, Italy \\ ${ }^{2}$ Università degli Studi e-Campus, Facoltà di Ingegneria Energetica, Novedrate (CO), Italy
}

\begin{abstract}
A built experimental set-up was employed for the estimation of the solid-liquid equilibria of alternative refrigerants systems. The behavior of carbon dioxide $+1,3,3,3$-tetrafluoropropene $\left(\mathrm{CO}_{2}+\right.$ $\mathrm{R} 1234 \mathrm{ze}(\mathrm{E})$ ) was measured down to temperatures of $160.0 \mathrm{~K}$. The results obtained for the mixtures were interpreted by means of the Schröder equation.
\end{abstract}

\section{Introduction}

For low temperature applications in industrial and commercial industry, cascade refrigeration cycles are a good option, especially if temperatures below $233.15 \mathrm{~K}$ are required [1]. Conventional cascade systems have been using HCFC, such as R13 (chlorotrifluoromethane, $\mathrm{CClF}_{3}$ ) and $\mathrm{R} 23$ (trifluoromethane, $\mathrm{CHF}_{3}$ ), as refrigerants, lately replaced by HFC. However, due to the increasing environmental concerns, several natural refrigerants, i.e. carbon dioxide, ammonia and nitrous oxide, have recently been considered for use in cascade refrigeration systems. In particular, various studies evaluated ammonia (R717) as the high-stage fluid and carbon dioxide (R744) as the low-stage fluid [2-5]. In this configuration, the lowest evaporator temperature achievable is limited by the melting temperature of carbon dioxide $\left(T_{m}=216.58 \mathrm{~K}\right)$, so that for those applications requiring temperatures below said limit, a solution is represented by blends of carbon dioxide with other lower boiling refrigerants: carbon dioxide + nitrous oxide, carbon dioxide + hydrofluorocarbons (HFCs), carbon dioxide + hydrocarbons (HCs) have been already considered as potentially suitable working fluids in lowtemperature refrigeration applications [6-8]. Other fluids that can be blended with $\mathrm{CO}_{2}$ in order to lower the evaporation temperature while keeping good environmental performance are the HydroFluoroOlefines (HFOs) R1234yf and R1234ze(E). They have been recently considered to replace traditional refrigerants thanks to their low GWP, that is 4 for R1234yf [9] and 6 for R1234ze(E) [10]. Particularly R1234yf is used for air conditioning applications, while $\mathrm{R} 1234 \mathrm{ze}(\mathrm{E})$ is receiving attention as refrigerant, blowing agent and aerosol. Moreover several attempts to use their blends to tailor traditional refrigerant for particular applications are under evaluations [11].

In this paper solid-liquid equilibria (SLE) for carbon dioxide and R1234ze(E) is experimentally investigated.
In fact, SLE data are important in refrigerating industry, defining the lowest temperature limit at which the refrigerant may circulates in fluid state and none information is available in literature for the considered mixtures. The same experimental apparatus has been used in the past to assess the SLE of several $\left(\mathrm{CO}_{2}\right.$ and $\left.\mathrm{N}_{2} \mathrm{O}\right)+$ HFCs binary systems [12-17] and the reliability of results obtained in such a way has been already proved.

\section{Experimental Set-up and Procedure}

The experimental setup is not reported here. More details can be found elsewhere [13].

The first step is the charging procedure, that is operated as it follows: the bottle containing the refrigerant gas is weighed on the electronic balance (the uncertainty of which is $0.5 \mathrm{mg}$ ); then the bottle is connected to the apparatus and to the vacuum pump (Vacuubrand, Mod. RZ2), used to create a vacuum inside the measuring cell; then the fluid is charged by opening the valve on the gas bottle; the temperature of the cell is brought down by a flow of liquid nitrogen so as to insert the whole mass in the cell, leaving as little as possible in the charging tube; a suitable time interval is allowed so that the pressure, being lowered by the temperature reduction, can drop to below atmospheric pressure, then the on/off valve is closed; and the gas bottle is disconnected and weighed again to establish the actual mass charged in the cell.

Once completed the charge, the cooling phase starts and by means of monitoring the time dependence of temperature, it is possible to draw a cooling curve for each sample concentration, containing the information about the solid-liquid transition. In fact, while the change of phase occurs, the heat removed by cooling is compensated for by the latent heat of the phase change, showing a change of slope in the temperature trend. The arrest in cooling during solidification allows the melting point of the material to be identified on the time- 
temperature curve. The melting points can then be plotted versus the composition to give a phase diagram.

All the uncertainties were calculated using the law of error propagation, as reported elsewhere [12]. Here, the previously reported results will be briefly summarized.

The total uncertainty of the mass of sample mixture was less than $0.01 \mathrm{~g}$. The uncertainty in composition measurements was estimated to be always lower than 0.005 in mole fraction.

The global uncertainty of the temperature, considering also the contribution of the Rossini method correction, was estimated to be less than $\pm 1 \mathrm{~K}$.

\section{Experimental results}

Measurements were taken using different concentrations of the two components, obtaining a satisfactory number of points, which were then recorded on a concentration/temperature graph $(T-x)$.

Conducting several tests on the same sample, we noted that we obtained better, more reliable results by switching off the stirrer at least about 40 to $50 \mathrm{~K}$ (values suggested by experience) before reaching the triple point temperature. This was probably due to the turning of the stirrer, that helps to keep the mixture's components well mixed and the homogeneity of temperature inside the cell, but once near-freezing temperatures have been reached, it probably interferes with the solidification of the mixture, fragmenting the solid crystals when they began to form.

In addition, conducting several tests on different sample compositions, better results were generally achieved when the apparatus was used in the cooling mode. When possible, however, data were collected with both configurations for the same concentrations and results were mutually consistent, with deviations always well within $1 \mathrm{~K}$. This value could be considered the reproducibility limit of our experimental measurements. This is confirmed by figure 1 , where the $T-x$ measurements for the system considered are reported. From the $T-x$ data, the eutectic point was detected (at around $x_{l}=0.30$ and $T=164 \mathrm{~K}$ ).

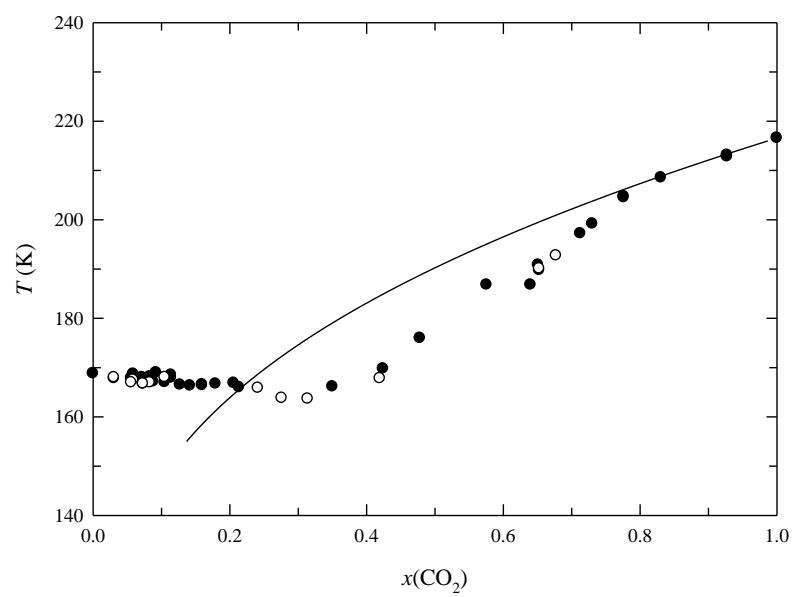

Figure 1. SLE for the $\mathrm{CO}_{2}+\mathrm{R} 1234 \mathrm{ze}(\mathrm{E})$ system. Open symbols denote the experimental points obtained by the heating mode, while black symbols denote the experimental points obtained by the cooling mode. The lines denote the Schröder eq.
Rossini method corrections. The results of the temperature data acquisitions were corrected using the Rossini method [13] because a constant cooling rate is not indispensable and was not guaranteed by our experimental method. The entity of the corrections takes into account the fact that the fluid is still in a liquid state during the metastable state (supercooling) that precedes proper solidification. In this phase, the temperature is distinctly lower than the one characterizing the instant when crystallization begins, its amplitude depending mainly on the rate at which the temperature is lowered. The resulting corrections were nonetheless always very limited, of the order of a few tenths of a Kelvin in the majority of cases, and always well below $1 \mathrm{~K}$.

\section{Interpretation of the results}

When an organic system forms eutectic and solidus is formed by pure system components, the course of the liquidus is well described by the Schröder equation [14]. The solubility of the solid solute in the solvent [here R1234ze(E)] can be described by the Schröder equation; which disregarding any difference between the heat capacity of the subcooled liquid solute and solid solute takes the following form:

$\ln \gamma_{2} x_{2}=-\frac{\Delta h_{\mathrm{m}}}{R T}\left(1-\frac{T}{T_{\mathrm{m}}}\right)$

where the subscript 2 denotes the solute and the subscript $\mathrm{m}$ denotes property at melting point. Assuming as a first approximation that the solute's activity coefficient, $\gamma_{2}=1$,we can write:

$\ln x_{2}=-\frac{\Delta h_{\mathrm{m}}}{R T}\left(1-\frac{T}{T_{\mathrm{m}}}\right)$

This simplification leads to the consideration that the solubility of the solid solute is independent of the solvent as far as the assumptions hold. The enthalpy at melting point $\left(\Delta h_{m}\right)$ was assumed to be $9020 \mathrm{~J} \cdot \mathrm{mol}^{-1}$ for $\mathrm{CO}_{2}[15]$ while for the solvents side, since no enthalpy data were available in the open literature for the HFOs, it was impossible to draw the prediction curve.

The course of the liquidus calculated with the Schröder equation is included in Figure 1.

For the studied system, excluding the very high $\mathrm{CO}_{2}$ concentrations $\left(x_{1}>0.8\right)$, the Schröder equation was clearly not able to describe the experimental result trend. In particular, very high discrepancies (up to $15 \mathrm{~K}$ ) were found at medium $\mathrm{CO}_{2}$ concentration $\left(0.2<x_{1}<0.8\right)$, and a significant shift of the eutectic point was revealed. The eutectic point was estimated at $x_{I}=0.30 \pm 0.05$ and $T=$ $164 \pm 1 \mathrm{~K}$. The composition of eutectic point, assuming that the system holds the Schroder equation, suggests an enthalpy of fusion of $\Delta h_{m}=17000 \mathrm{~J} \cdot \mathrm{mol}^{-1}$.

The observed deviations from the Schröder equation, if comparing results with the prediction using its simplified form expressed by equation (2), may come 
from various sources: $a$ ) Assumption that $\gamma_{2}(x)=1 ; b$ ) at very low temperatures, liquid phase is partially immiscible; $c$ ) solidus is not formed by pure components $\left(x_{1}=1\right.$, and on other branch $x_{2}=1$ ) because either they form mixed crystals or they are not pure, due to sideeffects as occlusion and/or inclusion.

However, the assumption $\gamma_{2}(x)=1$ seems to be realistic, as other $\mathrm{CO}_{2}+\mathrm{HFCs}$ systems showed an almost ideal in terms of the Raoult's law behaviour over a wide temperature range with no evidence in change of that trend with decreasing of temperature. For that reason, also eventual limited miscibility in liquid phase, even at low temperatures, should be rather not considered. In addition, the formation of solid solution, considering quite great differences in size and chemical nature of components, is less plausible. Concluding these speculations, it seems that the main contribution to the observed deviation from the Schröder equation is resulting from the mentioned above side-effects in solid phase formation. Of course, VLE data and system pressures measured over SLE experiment could give information for more precise interpretation of the observed deviation from the Schröder equation.

\section{Conclusion}

In this paper, the solid-liquid equilibria of carbon dioxide + 1,3,3,3-tetrafluoropropene $\left(\mathrm{CO}_{2}+\mathrm{R} 1234 \mathrm{ze}(\mathrm{E})\right)$ were measured down to temperatures of $160.0 \mathrm{~K}$. The binary system is potentially suitable working fluids in lowtemperature refrigeration applications, as cascade cycles. The measurements were performed both in the cooling and in the heating mode. The two configurations showed mutually consistent results.

For the $\mathrm{CO}_{2}+\mathrm{R} 1234 \mathrm{ze}(\mathrm{E})$ system, excluding the very high $\mathrm{CO}_{2}$ concentrations $\left(x_{1}>0.8\right)$, the Schröder equation was clearly not able to describe the experimental result trend, showing very high discrepancies at medium $\mathrm{CO}_{2}$ concentration $\left(0.2<x_{1}<0.8\right)$, and a significant shift of the eutectic point prediction.

\section{References}

1. W.F. Stoecker, Industrial refrigeration handbook, McGraw-Hill, New York, 1998

2. R. Taylor, Carbon dioxide-based refrigerant systems, ASHRAE J. 22, September 2002

3. H.M. Getu, P.K. Bansal, Int. J. Refrig. 3145 (2008)

4. S. Bhattacharyyaa, A. Garaia, J. Sarkarb, Int. J. Refrig. 321077 (2009)

5. J.A. Dopazo, J.F. Seara, J. Sieres, F.J. Uhía, Appl. Therm. Eng. 291577 (2009)

6. G. Di Nicola, M. Pacetti, F. Polonara, R. Stryjek, Proc. Int. Conf. on Refrig., Washington DC, USA, 2003

7. [7] G. Di Nicola, G. Giuliani, F. Polonara, R. Stryjek, Int. J. Refrig. 28130 (2005)

8. G. Di Nicola, F. Polonara, R. Stryjek, A. Arteconi, Int. J. Refrig., 34 (6) 1436 (2011)
9. O.J. Nielsen, M.S. Javadi, M.P. Sulback Andersen, M.D. Hurley, T.J. Wallington, R. Singh, Chem. Phys. Letters, 439 (1-3) 18 (2007)

10. R. Søndergaard, O. Nielsen, M. Hurley, T. Wallington, R. Singh, Chem. Phys. Letters, 443 (4-6) 199 (2007)

11. J.S. Brown, F. Polonara, G. Di Nicola, L. Fedele, S. Bobbo, C. Zilio, International refrigeration and air conditioning conference at Purdue, paper 2490, July 2012, West Lafayette, IN 47907-2032, USA, West Lafayette: Printing Services

12. G. Di Nicola, G. Giuliani, F. Polonara, R. Stryjek, J. Chem. Eng. Data 512209 (2006)

13. B.J. Mair, J.A.R. Glasgow, F.D. Rossini, J. Res. Nat. Bur. Standards 26591 (1941)

14. I. Schröder, Z. Phys. Chem. 11449 (1893)

15. R.L. Rowley, W.V. Wilding, J.L. Oscarson, Y. Yang, N.F.Giles, DIPPR ${ }^{\circledR}$ Data Compilation of Pure Chemical Properties, Design Institute for Physical Properties, AIChE, New York, 2010 